\title{
MOLECULAR EPIDEMIOLOGY AND FUNCTIONAL GENETICS OF MYCOBACTERIUM TUBERCULOSIS IN HUMANS AND ANIMALS
}

\author{
Muhammad Ahsan Naeem¹, Muhammad Younus², Qaiser Akram², Qamar-un-Nisa³ and Liu Zhenshan \\ 'Department of Basic Sciences, KBCMA College of Veterinary Animal Sciences, Narowal, 5160o, Pakistan \\ ${ }^{2}$ Department of Pathobiology, KBCMA College of Veterinary Animal Sciences, Narowal, 516oo, Pakistan \\ 32Department of Pathology, University of Veterinary Animal Sciences, Lahore, Pakistan \\ ${ }^{4}$ Key Laboratory of Zoonosis, Ministry of Education, Institute of Zoonosis, Jilin University, 5333 Xian Road, Changchun \\ 130062, Peoples' Republic of China
}

*Corresponding author: ahsan.naeem@uvas.edu.pk

\section{INTRODUCTION}

Tuberculosis (TB) is listed in the top 10 worldwide killing diseases of 21st century (WHO 2019). The global TB report 2019 had declared 10 million morbidities, 1.5 million mortalities and 0.484 million drug resistant TB cases in 2018 (WHO 2019). Mycobacterium tuberculosis (Mtb) is the causative agent of TB, which can form lesion(s) in any organ, such as lungs (Pulmonary TB) bones, lymph nodes, brain, kidneys and joints (extra-pulmonary TB) (Golden and Vikram 2005; Kumar et al. 2007; Rockwood 2007; Behera 2010). $M t b$ is an obligate intracellular bacterium of the family Mycobacteriaceae. Mycobacteria are non-spore forming, aerobic, straight rods or slightly curved and nonmotile. Colony morphology varies among species. The rod shaped (bacilli) appearance of $M t b$ by scanning electron microscopy (Fig. 1.1A) and the acid-fast staining of sputum sample (red rods) by light microscopy (Fig. 1.1B) can be observed. The characteristic features of the $M t b$ include: dormancy, slow growth complex cell envelope due to thick waxy coating and genetic homogeneity. The cell envelope of $M t b$ contains an additional layer beyond the peptidoglycan, which is highly rich in lipids, glycolipids and polysaccharides. Generation time of $M t b$, in growth medium or infected animals, is usually around 24 hours. Thus, the growth of mycobacterial species is slower compared to other bacteria. This slow growth of $M t b$ makes the TB disease chronic in nature.

Tuberculosis is an aerosol transmitted disease that spreads from infected population to healthy one (active TB) and may be sub-clinical i.e., does not show symptoms (latent/dormant TB). In the latent state, $M t b$ remains inactive within the infected tissue. As immunity decreases through ageing, poor diet, diabetes and HIV, the inactive bacteria become reactivated, causing an outburst of the disease. Active TB (ATB) has many communal symptoms which are low grade pyrexia, continuous coughing, blood spattered phlegm, weight loss, tiredness, chest pain and night sweats (Loddenkemper et al. 2015).

Tuberculosis can be over-come to some extent by Mycobacterium bovis BCG vaccination, which was developed and isolated by Calmette and Guérin in Lille, France (Calmette et al. 1927). The variable efficacy and response of BCG vaccine led to ineffective disease control (Andersen and Doherty 2005). The first anti-tuberculous drug (streptomycin) was developed during World War II by Selman Waksman (Waksman 1964). In the beginning, therapy with streptomycin seemed highly effective, but problems arose when resistance developed rapidly against this drug (Kaufmann and Parida 2007). In most cases, TB can be diagnosed and treated successfully with combination therapy of different antibiotics. However, total drug resistant (TDR), multidrug-resistant (MDR) and extensively drug resistant (XDR) tuberculosis remain a public health issue and a health security threat. World Health Organization estimated 60o,ooo new rifampicin resistant cases of TB (WHO 2018).

The genome of $M t b$ is rich with $\mathrm{G}+\mathrm{C}$ nucleotide pair (Bohlin et al. 2019). It is thought that progenitor of MTBC comprises of Mtb, M. bovis, M. bovis BCG, M. microti and M. africanum. This MTBC originated from soil bacterium, lacking inter-strain genetic diversity and nucleotide changes. The human $M t b$ is mainly originated from the bovine tubercle bacilli after domestication of cattle (Sreevatsan et al. 1997). Several studies have shown that $M t b$ can even infect bovines, though the severity of disease is less (Ameni et al. 2013). However, in biomedical research, $\mathrm{H}_{37} \mathrm{Rv}$ strain of $M t b$ has been used that has retained almost all the virulent features of clinical isolate. However, unlike clinical isolates, $\mathrm{H}_{37} \mathrm{Rv}$ is prone to genetic manipulation and anti-TB drugs. Shining rod shaped bacilli of $M t b$ have been shown in Fig. 1.

\section{History}

The origin of genus Mycobacterium has dated back to approximately 150 million years. Recent molecular techniques provided data of its origin in East Africa about 3 million years ago (Gutierrez et al. 2005) and current $M t b$ strains are believed to be instigated from these ancestors (Sreevatsan et al. 1997). Researchers have forecasted that main genetic variation among these strains was from 250 to 1000 years ago (Hirsh et al. 2004). The bacteriologist, Robert Koch, had claimed first time $M t b$ as the etiological agent of TB by delivering a lecture on Uber Tuberculosis on $24^{\text {th }}$ March, 1882 . In the past era, several names were designated to $\mathrm{TB}$, depending upon the clinical symptoms e.g., phthisis (health decline), scrofula (enlarged degenerated lymph nodes), consumption (severe weight loss), white plague (pallor skin in infected patients) and 
pott's disease (extra pulmonary tuberculosis affecting spine) (Daniel 2006). The earliest evidence of Mycobacterium tuberculosis infection dated back to 9000 years in a woman and infant buried in Eastern Mediterranean (Philipp et al. 1996).

\section{Transmission and Pathogenesis}

A thorough understanding of $M t b$ transmission is necessary for the development of effective TB control policy. As TB is an aerosol transmissible disease, inhalation is the main portal of transmission. Multiple factors are also involved in its transmission e.g. immune status of the person exposed to infection, stage of infectiousness, as severely infected patients shed more tubercle bacilli, and period of exposure. Aerosol droplets (1-5 $\mu$ in diameter) transmit mycobacteria through sneezing, spitting, coughing and speaking (Cole et al. 1998; Long and Schwartzman 2014). Around 40,000 droplets have been discharged in a single sneeze. Each droplet is considered as a disease transmitting moiety on its inhalation (Klettner et al. 2012). Most of the times, infection is subclinical and the disease persists in dormant form (Cheigh et al. 2010). Yates et al. (2016) have demonstrated the transmission cycle of $M t b$ and a schematic figure has been drawn by taking concept from their paper and is shown in Fig. 1.2a.

The main feature behind effective pathogenesis of $M t b$ is its biological adaptation in a highly mutable environment and its slow growth under in vitro and in vivo conditions. Both impart its survival within macrophages necessary for the infection development (Bhardwaj 2014). The infection commences when $M t b$ enters alveolar macrophages and replicates within its phagosome (Kumar et al. 2007). Macrophages crack to eradicate $M t b$ via phagocytosis by considering it as a foreign entity. This happens by the fusion of phagosome with lysosome to form phagolysosome, which is an acid-killing trap for $M t b$. On the other hand, a thick waxy capsule around $M t b$ shields it from noxious elements. The bacterium multiplies within macrophage till bursting of the immune cell and dissemination of infection throughout the body. A recent research has described that a mutant $M t b$ strain, by knocking out of Rv3167c (a transcription factor), overexpresses phthiocerol dimycocerosates (PDIM), which is an $M t b$ virulent factor and endorses necrosis, resulting in phagosome mediated escape of $M t b$ (Quigley 2017). Delogu et al. (2013) have described the pathogenesis of TB and a schematic diagram has been plotted by taking concept from their research study, which is shown in Fig. 1.2b.

\section{Epidemiology and Topographical Spread}

Tuberculosis is a poverty associated disease and most commonly affects young individuals (Glaziou et al. 2015). Recent TB statistics demonstrates that around 10 million people got $\mathrm{TB}$, of which 3.2 million (32\%) women, 1.2 million (12\%) children and 5.6 million (56\%) men are diagnosed with this vicious infection (WHO 2019). Unemployment, food insecurity, psychological circumstances, poor housing conditions, illiteracy and congested social setups are the main aspects in high load TB countries (WHO 2014a). A total of 95\% deaths, reported in $3^{\text {rd }}$ world countries, are caused by TB (WHO 2014a). Persons with human immunodeficiency syndrome (HIV) or cases of other immunocompromised anomalies, such as silicosis, renal insufficiency and diabetes, are more susceptible to tuberculosis infection (Glaziou et al. 2013). However, a universal drop in TB cases was detected by the induction of direct observation treatment strategy (DOTS) and increasing the therapeutic consequences for recurred and newly emerged TB cases. In the recent global TB report of WHO, the data have been collected from different regions of continents and presented in Table 1.3 of this chapter, courtesy of Table 8.5 of "The global TB database 2020" (https://apps.who.int/iris/bitstream/handle/10665/ 336069/9789240013131-eng.pdf).

Tuberculosis has caused more deaths than human immunodeficiency syndrome virus (HIV) in 2015. Around $60 \%$ of high TB burdened areas are China, Pakistan, India, Indonesia, South Africa and Nigeria. Especially, the highly populated and distressing part of world for TB infection is the South East Asian region with maximum number of reported MDR-TB cases (with 54\% treatment coverage). African regions have $39 \%$ HIV positive TB patients and $48 \%$ overall treatment coverage. American, Western and European regions are provided with maximum treatment coverages, which are 81,84 and $78 \%$, respectively. Global epidemiology of tuberculosis and its progress towards achieving global targets has been presented by the Center of Disease Control and prevention $(C D C)$. The map is taken from the morbidity and mortality weekly report (MMWR) of the Center for Disease Control and Prevention (CDC) and shown in Fig. 1.3 (MacNeil et al. 2019).

The $M t b$ can also cause TB in animals, including reptiles, rodents, birds and elephants. In animals, almost all the ruminants and non-ruminants' species can be infected with bovine TB. An interesting case report demonstrated the TB cross-transmission from animal to human and back to animal. The pathogenic bovine TB strain was isolated from the cattle, which got infection from humans. Actually, this person got bovine TB bacterial infection through exposure in his childhood (Fritsche et al. 2004). Tubercle Bacilli are shed almost in every secretion and excretion, such as respiratory tract secretions, milk, feces, tears, urine and other body fluids of infected animals.

Bovine TB is caused by M. bovis, with similar presentation of lesions is observed in pulmonary and extra-pulmonary level, just like humans. In cattle, $M t b$ takes entry through the inhalation route, however, the activity level of infective organism depends on the health of immune status. In case of suppressed immunity, the infection disseminates to the extra-pulmonary organs like spleen, kidneys, liver and lymph nodes and this is known as military TB. Debilitation, emaciation or general body weakness, loss of weight, low grade fever and anorexia are general bovine TB symptoms (Ayele et al. 2004). Even researches have declared that eating dairy products of milk from infected animal, or drinking raw milk of such animal can also transmit bovine TB to humans (Davies 2006). 
Table 1.3: Status of core elements of multi-sectoral accountability in 2020 for 30 high TB burden countries, WHO regions and globally (Global TB database 2020)

a) National strategic plan (NSP) for TB and annual TB report

\begin{tabular}{|c|c|c|c|c|c|c|c|c|c|}
\hline $\begin{array}{l}\text { High TB burden } \\
\text { countries and } \mathrm{WHO} \\
\text { regions }\end{array}$ & $\begin{array}{l}\text { Number of } \\
\text { countries and } \\
\text { territories }\end{array}$ & NSP E & & $\begin{array}{l}\text { Represent } \\
\text { societies a } \\
\text { communi } \\
\text { involved i }\end{array}$ & $\begin{array}{l}\text { ivil } \\
\text { d } \\
\text { ctively } \\
\text { elopment }\end{array}$ & $\begin{array}{l}\text { NSP wa } \\
\text { updated } \\
\text { high lev } \\
\text { TB in Se }\end{array}$ & $\begin{array}{l}\text { ped or } \\
\text { he UN } \\
\text { ing on } \\
\text { r } 2018\end{array}$ & $\begin{array}{l}\text { Annua } \\
\text { availab }\end{array}$ & $\begin{array}{l}\text { report } \\
\text { blicly }\end{array}$ \\
\hline High TB burden countries & 30 & 30 & $100 \%$ & 29 & $97 \%$ & 25 & $83 \%$ & 27 & $90 \%$ \\
\hline Africa & 47 & 42 & $89 \%$ & 40 & $85 \%$ & 32 & $68 \%$ & 39 & $83 \%$ \\
\hline The Americas & 45 & 32 & $71 \%$ & 21 & $47 \%$ & 16 & $36 \%$ & 21 & $47 \%$ \\
\hline Eastern Mediterranean & 22 & 17 & $77 \%$ & 11 & $50 \%$ & 12 & $55 \%$ & 16 & $73 \%$ \\
\hline Europe & 54 & 25 & $46 \%$ & 21 & $39 \%$ & 14 & $26 \%$ & 30 & $56 \%$ \\
\hline South-East Asia & 11 & 11 & $100 \%$ & 9 & $82 \%$ & 8 & $73 \%$ & 9 & $82 \%$ \\
\hline Western Pacific & 36 & 21 & $58 \%$ & 14 & $39 \%$ & 15 & $42 \%$ & 19 & $53 \%$ \\
\hline Total & 215 & 148 & $69 \%$ & 116 & $54 \%$ & 97 & $45 \%$ & 134 & $62 \%$ \\
\hline
\end{tabular}

b) High-level review mechanism(s)

\begin{tabular}{ll}
\hline $\begin{array}{l}\text { High TB burden } \\
\text { countries and WHO } \\
\text { regions }\end{array}$ & $\begin{array}{l}\text { Number of NSP Exits } \\
\text { countries and } \\
\text { territories }\end{array}$
\end{tabular}

\begin{tabular}{|c|c|c|c|c|c|c|c|c|c|}
\hline $\begin{array}{l}\text { High TB burden } \\
\text { countries and WHO } \\
\text { regions }\end{array}$ & $\begin{array}{l}\text { Number of } \\
\text { countries and } \\
\text { territories }\end{array}$ & NSP E & & $\begin{array}{l}\text { Represen } \\
\text { societies } \\
\text { communi } \\
\text { involved i }\end{array}$ & $\begin{array}{l}\text { ivil } \\
\text { d } \\
\text { ctively } \\
\text { lopment }\end{array}$ & $\begin{array}{l}\text { NSP wa } \\
\text { updatec } \\
\text { high lev } \\
\text { in Septe }\end{array}$ & $\begin{array}{l}\text { ped or } \\
\text { le UN } \\
\text { ng on TB } \\
18\end{array}$ & $\begin{array}{l}\text { Annua } \\
\text { availal }\end{array}$ & $\begin{array}{l}\text { report } \\
\text { dblicly }\end{array}$ \\
\hline High TB burden countries & 30 & 16 & $53 \%$ & 12 & $40 \%$ & 15 & $50 \%$ & 7 & $23 \%$ \\
\hline Africa & 47 & 26 & $55 \%$ & 24 & $51 \%$ & 22 & $47 \%$ & 11 & $23 \%$ \\
\hline The Americas & 45 & 13 & $29 \%$ & 6 & $13 \%$ & 8 & $18 \%$ & 2 & $4 \%$ \\
\hline Eastern Mediterranean & 22 & 6 & $27 \%$ & 3 & $14 \%$ & 5 & $23 \%$ & 1 & $5 \%$ \\
\hline Europe & 54 & 19 & $35 \%$ & 14 & $26 \%$ & 16 & $30 \%$ & 7 & $13 \%$ \\
\hline South-East Asia & 11 & 7 & $64 \%$ & 4 & $36 \%$ & 6 & $55 \%$ & 3 & $27 \%$ \\
\hline Western Pacific & 36 & 15 & $42 \%$ & 11 & $31 \%$ & 12 & $33 \%$ & 6 & $17 \%$ \\
\hline Total & 215 & 86 & $40 \%$ & 62 & $29 \%$ & 69 & $32 \%$ & 30 & $14 \%$ \\
\hline
\end{tabular}

\section{Reverse Zoonosis: Mtb Infection in Bovines}

Humans also serve as the potential source for TB transmission to animals via reverse zoonosis (Hackendahl et al. 2004; Messenger et al. 2014). Transmission of Mtb to grazing cattle in Central Ethopia was through a traditional practice of spitting crushed tobacco from farmer's mouth directly into grazing cattle, which is a common practice and was considered the main route of $M t b$ transmission in cattle. 16srRNA locus sequence of isolates was used for differentiating the non-tuberculous mycobacteria (NTM) from the tuberculous mycobacteria (TM) (Ameni et al. 2011). TM were then used for region of difference (RD) typing, a standard method of differentiation TM at species level (Parsons et al. 2002). The RDs (RD4, 9 and 10) were selected by designing their primers for PCR analysis and declared $27 \%$ isolates were Mtb (Brosch et al. 2002; Berg et al. 2009). It was also concluded that $M t b$ infection in cattle mostly occurred in high human TB burden areas (Ameni et al. 2011).

Transmission of $M t b$ from human to cattle was through inhalation of cough droplets of active pulmonary TB patients or the ingestion of their sputum/urine contaminated pasture. The interesting point reported was less virulent form of disease in cattle (Ameni et al. 2013). From Ameni et al. (2013) studies, spoligotyping pattern of MTBC species and its schema of image has been drawn and shown in Fig. 1.4a.

Three isolates of $M$. bovis were isolated from two oxen of a farmer with active pulmonary tuberculosis. These three isolates had the same spoligotype pattern and were SB1176. The other two isolates were $M t b$ and were from cattle owned by farmers with active tuberculosis. These isolates had different spoligotype pattern and were SIT149 and SIT53.

The study of Villarreal-Ramos et al. (2018) has experimentally supported the claim of Ameni et al. (2013) reports regarding attenuation of $M t b$ in cattle (VillarrealRamos et al. 2018). Villarreal-Ramos et al. (2018) have suggested bovine model as one health approach for new TB biomarkers/vaccine candidates' development. RNAseq/transcriptome profiling was done from whole blood of $M$. bovis AF2122/7 and Mtb $\mathrm{H}_{37} \mathrm{Rv}$ infected cattle $(\mathrm{n}=4)$ at day 14 and 42 . Top 10 differentially expressed genes in animals at 14 and 42 days by infecting with $M$. bovis and $M t b$ have been shown and intrigued below in Fig. 1.4b by considering the findings from Villarreal-Ramos et al. (2018) studies.

M. bovis (AF2122/97) and Mtb $\mathrm{H}_{37} \mathrm{Rv}$ strains were used for infection. For transcriptional analysis, first 10 differentially expressed genes (DEGs) were studied at 14 and 42 days of infection (FDR $<0.05)$. $\log _{2}$ fold change $\left(\log _{2} \mathrm{FC}\right)$ values are shown on $y$-axis of the graph for the selected genes which means the relative expression of each gene in $M$. bovis infected animals to $M t b$ infected animals and vice versa. The $\mathrm{x}$-axis represents the days at which this study was conducted.

\section{Vaccines for TB}

The reasonable, resourceful and effective way of controlling any disease in a population is through vaccination (Khatoon et al. 2017). The whole organismbased vaccines (attenuated and killed) have a drawback of 




Fig. 1: Shining rod shape bacilli of Mtb (courtesy by Dr. Tahir Rasool, DHQ Hospital, Faisalabad): Arrow head depicts truant auramine-rhodamine (AR) stained acid-fast $M t b$ bacilli by using fluorescent microscope. The slide was prepared from the sputum sample of a TB positive patient.

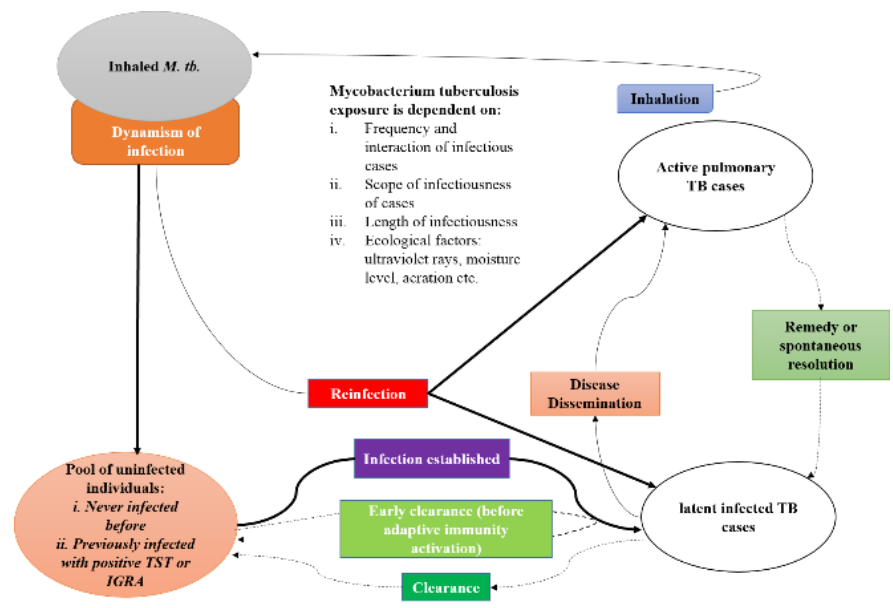

Fig. 1.2a: Schematic illustration of $M t b$ transmission cycle

containing non-antigenic potential fractions. Being the most widely used vaccine, BCG has been given over 4 billion times. However, BCG can induce a disseminating disease in individuals with mutation in immune modulating genes and infants with clinically active HIV (Hesseling et al. 2007). Moreover, BCG can be reverted back to virulent form, so the vaccine is not effective for worldwide TB control. BCG is a weakened strain of Mycobacterium bovis, which was developed between 1906 and 1919. It is still in use and is the only available vaccine against TB. This vaccine can prevent the neonates and children from the spread of TB and mortality. However, the use of BCG vaccine has some serious limitations and its efficacy is still questionable. The controversies about BCG include: i) fails to provide protection against TB in adults i.e. pulmonary $\mathrm{TB}$, ii) fails to provide protection

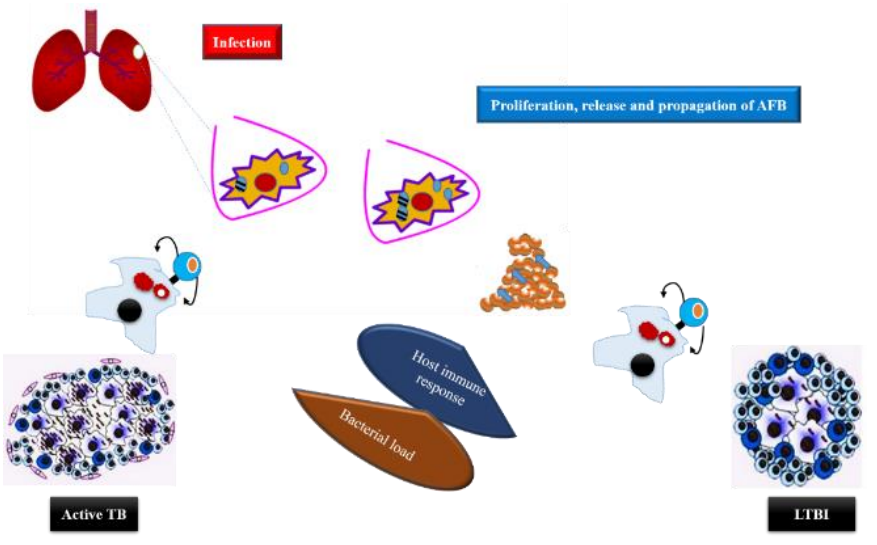

Fig. 1.2b: Pathogenesis of tuberculosis: Figure represents the aerosol inhalation of $M t b$ which then move in lungs, where they encounter with the host innate immune system (alveolar macrophages). If the $M t b$ overcome macrophages, they start multiplying and disseminate to other tissues (military $\mathrm{TB}$ ) via blood and lymphatic streams. Only the T cell immunity (cellmediated) can undermine the infection and in $>90 \%$ cases overt signs of $\mathrm{TB}$ become covert (Latent TB). However, if cell mediated immunity is subverted by somehow, then active bacterial replication starts and heavy tissue damage occurs (active TB) (Delogu et al. 2013).

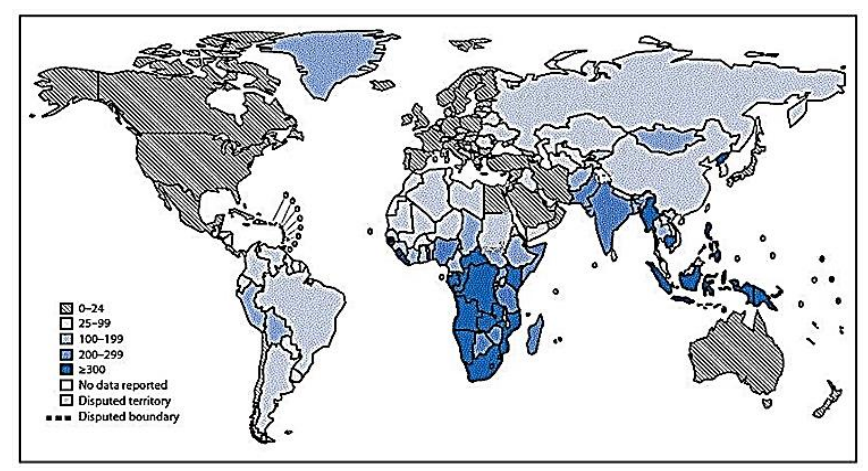

Fig. 1.3: Annual TB incidences (per 100,000 population), by region- worldwide, 2017 (MacNeil et al. 2019)

against chronic $\mathrm{TB}$, iii) has no booster for repeating vaccine and, iv) difficulty in diagnosis of actually diseased and vaccinated individuals by common diagnostic test e.g. tuberculin skin test (Crampin et al. 2009; Glick et al. 2014). Consequently, $M t b$ has undergone to dormant state in most cases after BCG vaccination and latent chronic infection has been established. The latent $M t b$ becomes active when the strong immune status of person becomes weakened e.g., individuals with genetic immune defects, aged people and immunosuppressant drugs taking people. Thus, there is a strong need to develop vaccines that can either act as BCG's booster or substitute the BCG (Hesseling et al. 2007).

Moreover, to overcome these issues of BCG, an alternative or ancillary vaccine is essential which is safer, more immunogenic and induces longer lasting protection. An answer could be peptide vaccine(s) that identify the peptide epitopes on immunogens to generate the immunogenic response. For this purpose, synthetic versions of peptide epitopes have been used by engineering the vaccine. Contrary to customary vaccine, 
peptide vaccines are non-infective, totally synthetic, zero risk of pathogen activation and free from unwanted effects, which are common while using whole organism based attenuated vaccine(s) (Glick et al. 2014).

Currently, 12 vaccines for TB are in different phases of clinical trial (Zhu et al. 2018). These could be weekend pathogenic strain, viral vaccine, recombinant BCG vaccines, DNA vaccines and subunit/peptide vaccines (Kaufmann 2014; Zhu et al. 2018). The concept of peptide vaccine has been aroused during the past one or two decade(s). The reason is to generate non-infective and broad-spectrum vaccine by using only immunogenic part of organism instead of using full organism. These subunit vaccines contain only the immune system stimulatory part of pathogen. Currently, these vaccines are becoming more precise due to the use of only a high binding affinity epitope, instead of using full peptide, that can stimulate the production of antibodies or pro-inflammatory and inflammatory cytokines (Glick et al. 2014; Bellini and Horváti 2020; Gong et al. 2021). Since subunit vaccines contain only the essential part of antigens, the risks of adverse reaction to the vaccine are lower. Firstly, it has to be effective, and an epitope (antigenic determinant) must constitute a short stretch of adjoining amino acids. Secondly, the peptide vaccine must have the same $3 \mathrm{D}$ conformation as its isolated epitope in the intact viral particle. Conventional vaccines have undesirable antigens that have no or little contribution in providing protective immunity and also these antigens sometimes complicate the situation by inducing atopic responses. Peptide vaccines are an attractive alternative strategy that relies on the use of short peptide fragments to engineer the induction of highly targeted immune responses. While talking about peptide vaccines, both $\mathrm{CD}_{4}+$ and $\mathrm{CD} 8+\mathrm{T}$ cell epitopes are presented on the surface of an antigen presenting cell (APCs) for binding with MHC molecules. For designing peptide vaccines, databases are available for the prediction of $\mathrm{T}$ and $\mathrm{B}$ cell epitopes. The accurate selection of these epitopes is necessary, so that the shortlisted epitopes could have a strong binding affinity with MHC molecule for eliciting immune response (Glick et al. 2014; Bellini and Horváti 2020; Gong et al. 2021).

\section{Peptide/subunit Vaccines for TB}

Subunit vaccines were designed on immunoinformatic logics and evaluated by in silico analysis that provides confidence for their in vitro and in vivo analyses. In the study of Dhivya et al. (2018), T cells epitopes were obtained from polyketide and non-ribosomal peptide synthesis proteins of $M t b$. These epitopes were used as potential vaccine candidates for designing vaccine by immunoinformatics approach. Polyketide and nonribosomal proteins belong to the class of small molecule metabolites of $M t b$, used in bacterial envelope organization, virulence, and pathogenesis. A total of 41 proteins from both classes of proteins were analyzed computationally for obtaining possible overlapping peptides. Similarly, in another research work (Hossain et al. 2017), a computationally identified and characterized
Mtb Ag85B T-cell epitopes-based subunit vaccine was designed. Computational tools for generating T-cell epitopes predicted an epitope, 181- QQFIYAGSLSALLDP195, that could bind to at least 13 MHC molecules, demonstrating the widened nature of epitope. Moreover, these workers also analyzed the "Allele Frequency Database," for the spread of HLA alleles throughout worldwide population and found that both HLA-I \& II alleles are frequently present in TB- endemic regions (Hossain et al. 2017).

Choi et al. (2018) used the concept of subunit vaccine against $M t b$ in which ESAT-6 protein was combined with $\mathrm{PE} / \mathrm{PPE}$ protein $\mathrm{CD}_{4}+$ epitope. Then this combination was used against HN878- highly virulent strain of $M t b$. In response to that, $\mathrm{CD}_{4}+$ cells of mice released copious amount of IFNr and IL-2 i.e., the cytokines considered protective against $M t b$ infection. Moreover, CFU count of $M t b$ in spleen was also found reduced in this study. This means that this subunit vaccine works well against virulent $M t b$ strain (Choi et al. 2018).

Another subunit vaccine $\mathrm{H}_{56 / \mathrm{CAF} 1}$ was tested against $M t b$. The study revealed that $\mathrm{CD}_{4+} \mathrm{T}$ cells memory generated by $\mathrm{H}_{56} / \mathrm{CAFo1}$ vaccination was lost in natural infection of $M t b$. The possible reason was the changes in ESAT- 6 and Ag85B proteins of $M t b$ in lungs. This leads to high expression of KLRG1 expression on $\mathrm{CD}_{4}+\mathrm{T}$ cells surface and decreases the production of TNF $\alpha$ and IFNr. This subunit vaccine, however, provides excellent protection against $M t b$ re-infection with excessive amount of IL-1 and IL-17A production. Both these cytokines provide protection to counter TB infection (Lindenstrøm et al. 2017).

One more subunit vaccine was designed and tested according to the infection states of $M t b$, such as active, latent and reactivation. In all these states, metabolic states of bacteria were variable. In this work, single-, two-, poly- and multi-stage vaccines were developed against TB infection and tested in mice. The results showed that multi-stage vaccine provided more immunity than all others and this was due to the detection of IFNr and IL-2 producing $\mathrm{CD}_{4}+\mathrm{T}$ cells in mice (Ma et al. 2017).

\section{Genomic Organization Features}

The genome of $M t b$ was studied generally using the $\mathrm{H}_{37} \mathrm{Rv}$ strain. A total of 4,411,529 base pairs (bp) are present in $M t b$ genome rich in $\mathrm{G}+\mathrm{C}$ contents of approximately $65.6 \%$. High $\mathrm{G}+\mathrm{C}$ content in $\mathrm{Mtb}$ genome is apparent with highest percentage of GTG initiation codons of $35 \%$ compared to $9 \%$ in Bacillus subtilis and $14 \%$ in E. coli (Cole et al. 1998). An integrated genomic map of 4.4 mega base (Mb) in circular form has been shown in Fig. 1.7, the resource is copied from Tuberculist open access web server and ordered libraries of cosmids and bacterial artificial chromosomes (BACs) (Philipp et al. 1996; Brosch et al. 1998). Mtb genome is also rich in repetitive DNA (insertion/transposon) sequence. The insertion sequences in $M t b \mathrm{H}_{37} \mathrm{Rv}$ genome are mostly slot in to the intergenic regions. Around 3,924 open reading frames (ORF) are present in genome, constituting $\sim 91 \%$ 


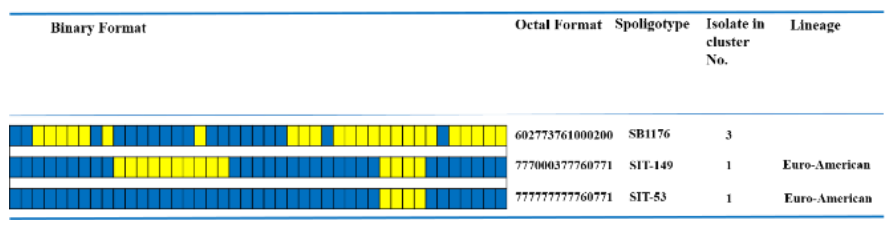

Fig. 1.4a: Spoligotype patterns of MTBC species (M. bovis isolates from Bos Taurus).

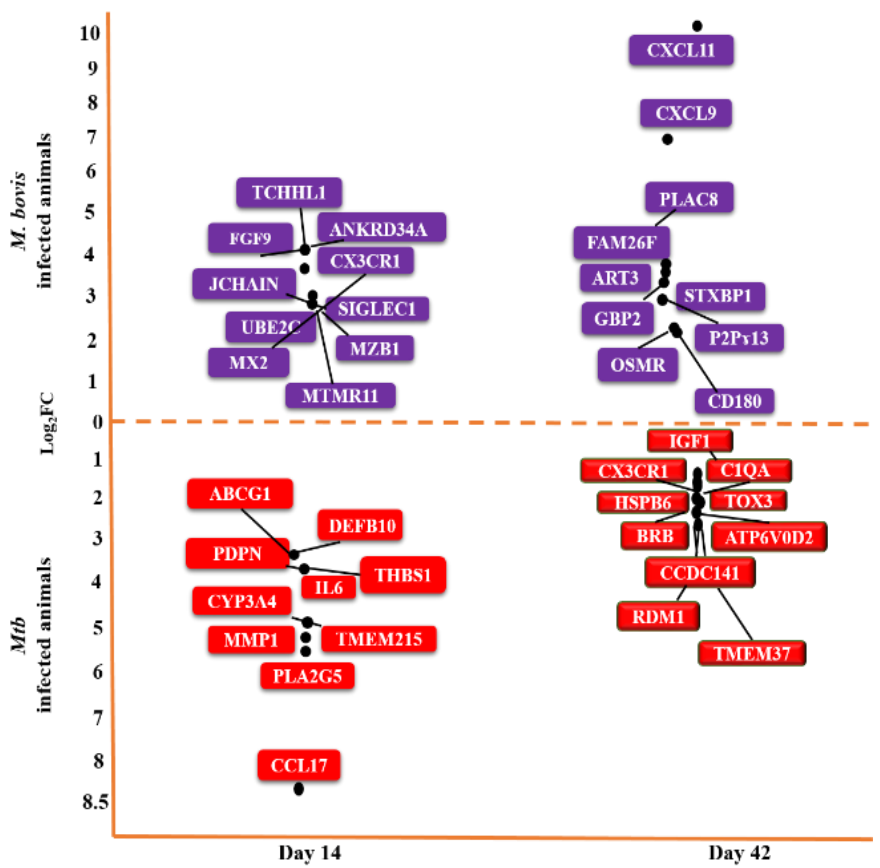

Fig. 1.4b: $M$. bovis or $M t b$ infection mediated transcriptome analysis (RNA sequencing), using whole blood non-activated sample.

of coding capacity. On the other hand, virulence transfer by innate plasmids of $M t b$ is quite easy than chromosomal gene transfer. Moreover, Mtb genome encodes around 190 transcriptional regulators, which include: 11 two-component system, 13 sigma factors and more than 140 other transcription regulators.

\section{Functional Genetics of $M t b$}

\section{Nuclear Localized Proteins}

Nuclear localized proteins have nuclear localization signal (NLS) to move inside host nucleus and make chromosomal conformational changes. Because of these changes, these proteins are sometimes called nuclear modulators. Rv2966c is a nuclear localized protein of $M t b$ that interacts with host chromatin through non-CpG methylation and has histone $\left(\mathrm{H}_{3} \& \mathrm{H}_{4}\right)$ binding. Rv2966c localizes to the nucleus and then binds to DNA sequences of host chromatin, methylates cytosines in the non-CpG region. They have also described the significance of their study in a sense that it was the first one to explore such a protein of $M t b$ that can methylate host DNA in noncanonical manner (Sharma et al. 2015). Similarly, Rv1988 protein was discovered to influence the host genome by methylating the non-tail arginine of histone $\mathrm{H}_{3}$. Rv1988 is

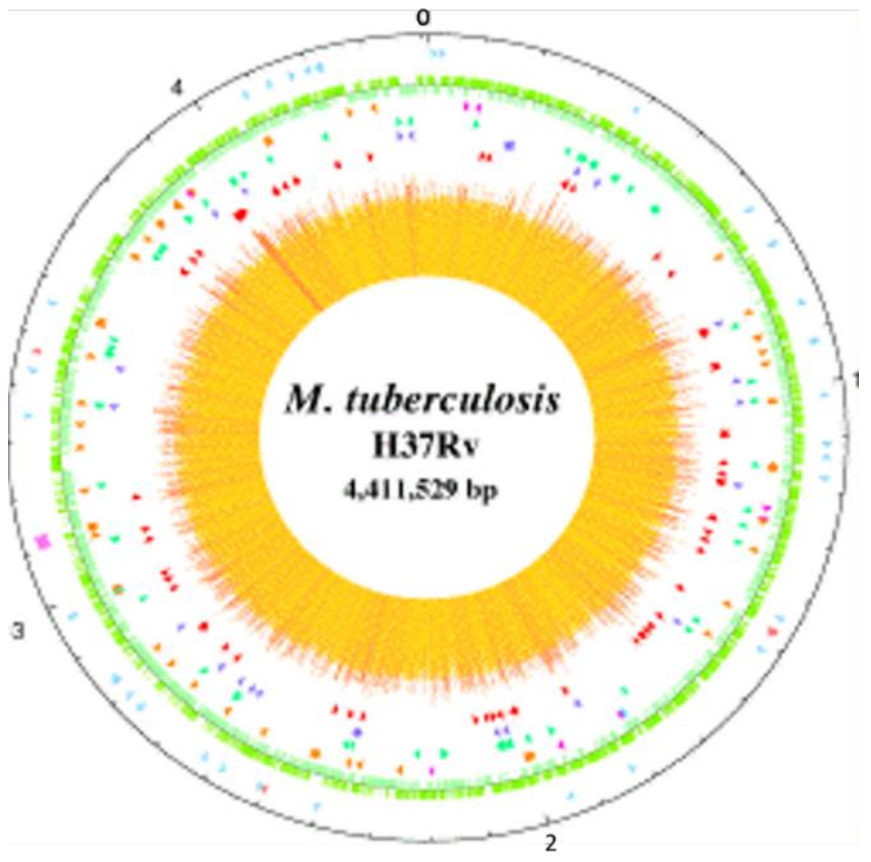

Fig. 1.7: Circular representation of the $M t b$ chromosome illustrating the location of each gene/protein-coding in $\mathrm{H}_{37} \mathrm{Rv}$ strains: The figure is taken from Tuberculist open access web (http://genolist.pasteur.fr/TubercuList/) server. The image depicts the full genomic map of $M t b$ chromosome, including; protein coding, non-coding, synonymous or non-synonymous substitutions and IS611o elements insertion.
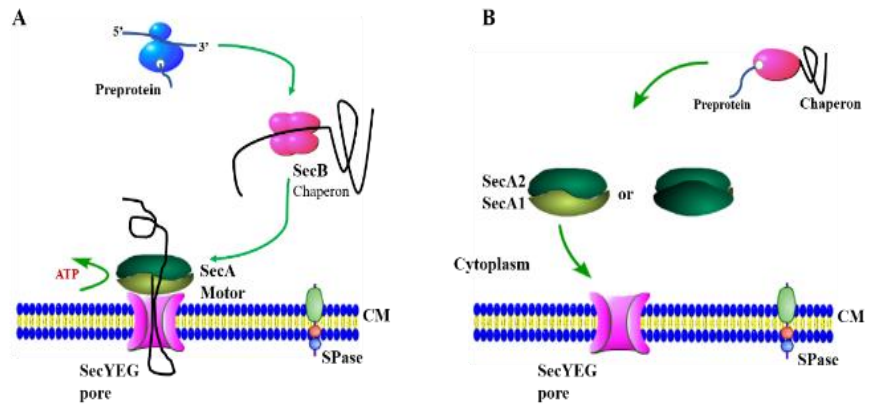

Fig. 1.8.2a: Model of protein secretion: A) It shows the canonical secretion pathway of Sec system. Mtb ribosome (blue) synthesized a pre-protein and delivered to chaperon which is SecB (a complex type of protein- pink). This SecB maintains the protein in folded form and passed on to SecA. SecA is complex (light/dark green) that acts as motor by consuming ATP. SecA binds with SecYEG pore and then hydrolyse ATP and use its energy to deliver the protein to SecYEG. SecYEG acts as cell membrane pore through which formed protein can go out for functioning. B) In this suggested model, instead of SecB, just chaperon is a protein to maintain the folded protein and deliver it to SecA. Here SecA is in complex form as a heterodimer (SecA1/SecA2). Both after complexing uses ATP and bind on SecYEG pore to deliver out the synthesized protein from $M t b$ cell. CM- cell membrane, SPase-signal peptidase (Prabudiansyah et al. 2015)

an outgoing (secreted) mycobacterial protein that is functionally characterized as methyltransferase and localized to the nucleus of host cell to interact with host chromatin material. Rv1988 methylated histone $\mathrm{H}_{3}$ at arginine 42 position $\left(\mathrm{H}_{3} \mathrm{R}_{42}\right)$ and suppressed the expression of genes involved in the defense against $M t b$. 


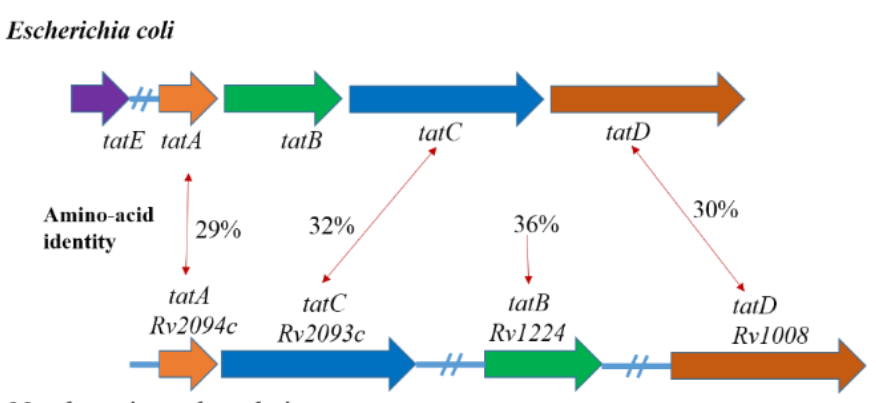

Mycobacterium tuberculosis

Fig. 1.8.2b: Organization of Mycobacterium tuberculosis TAT genes: Comparative analysis of genetic arrangement of TAT genes between $M t b$ vs $E$. coli. Percent identity of the respective homologous for TAT proteins has been shown. A $16 \mathrm{bp}$ intergenic region has separated the TATA and TATC genes in Mtb (Saint-Joanis et al. 2006).

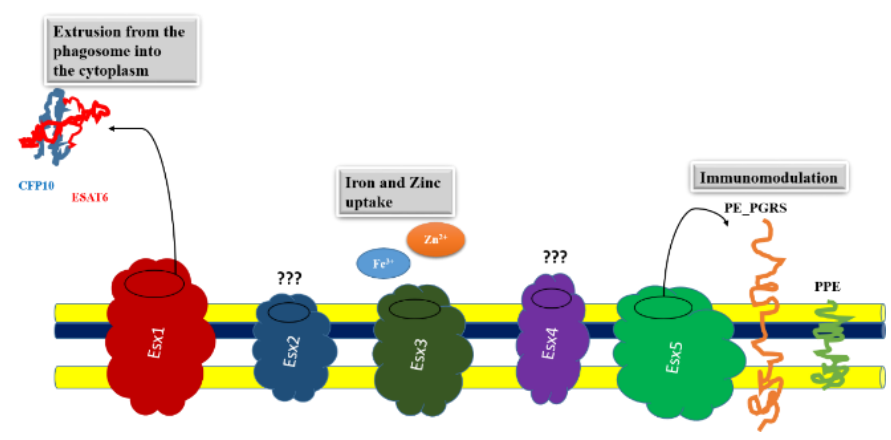

Fig. 1.8.2c: Esx $\left(\mathrm{T}_{7} \mathrm{SS}\right)$ Secretion system(s): Five components have been shown schematically. Esxı is involved in phagosomal lysis within macrophages that liberate $M t b$ within cellular cytoplasm. Esx3 is involved in iron and zinc uptake or homeostasis. Esx 5 gives growth to bacteria. Esx2 and 4 function is still in exploration (Delogu et al. 2013).

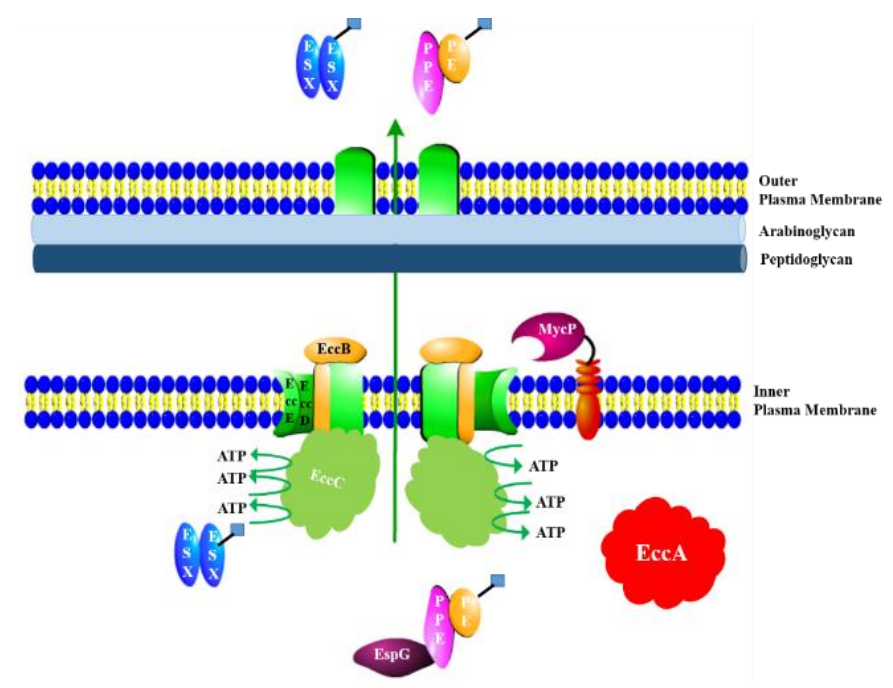

Fig. 1.8.2d: Proposed model of $M t b$ special type 7 secretory system $\left(\mathrm{T}_{7} \mathrm{SS}\right)$. Ecc- ESX conserved components.

A non-tail histone methylation at arginine 42 position ( $\left.\mathrm{H}_{3} \mathrm{R}_{42} \mathrm{me}_{2}\right)$ happened at the entry and exit point of DNA in the nucleosome. However, within regulatory sites in the $\mathrm{N}$-terminal tail, this methylation is not present. Knock out of Rv1988 in Mtb decreases bacterial survival in the host, and experimental expression through plasmid cloning of Rv1988 in non-pathogenic Mycobacterium smegmatis adversely affects the infected mice health. Thus, it can be inferred that Rv1988 is an important growth regulatory virulence factor and uses noncanonical pathway to regulate host cell transcription (Yaseen et al. 2015). Rv3423 is another nuclear localized protein, acting as histone acetyltransferase. Bioinformatics predictions showed that Rv3423 acetylates histone $\mathrm{H}_{3}$ at lysine $\mathrm{K}_{9}$ and $\mathrm{K}_{14}$ positions. The protein is necessary for intracellular growth of bacteria. The histone acetyltransferase activity of $\mathrm{Rv}_{3423}$ influences the expression of host anti-inflammatory genes to inhibit bacterial clearance and to promote intracellular survival of bacteria (Jose et al. 2016). Mycobacterial phosphatase PtpA, which acts in the host cytoplasm, can also localize to host nucleus and control the expression of host cell proliferation controlling genes. PtpA is an effector protein of $M t b$, secreted outside the bacterial cell and regulates the immune regulatory proteins, such as $\mathrm{p}-\mathrm{p} 38, \mathrm{p}-\mathrm{VPS}_{33} \mathrm{~B}$ and $\mathrm{p}-\mathrm{JNK}$, by dephosphorylating them in the host cytoplasm. Hence, it suppresses the host innate immunity. The most significant host gene controlled by PtA is GADD 45A i.e. PtpA that can bind directly to the GADD 45A promoter (Wang et al. 2017).

\section{Secretory systems}

Currently, much of the data are available on $M t b$ genomics and proteomics. Pathogenesis of TB is largely dependent on secretory (exported) proteins of $M t b$. These proteins are exported into host cell(s) and modulate its functions, especially those related to immunity (Majlessi et al. 2015). Three main secretory systems are present in $M t b$, which are involved in exporting their secretory proteins to the external environment (DiGiuseppe and Cox 2007). These include: general secretory (Sec) system, twin arginine translocation (TAT) system and Type VII secretory system $\left(\mathrm{T}_{7} \mathrm{SS}\right)$ or Esx secretory system. Sec system has Sec A, D, E, F, G and Y proteins, which are unfolded in nature with $\mathrm{N}$-terminal signal sequence across the bacterial cell membrane (DiGiuseppe and Cox 2007). SecA2 (member of SecA) has great importance in imparting virulence to $M t b$ (Lenz et al. 2003). Sec system, the diagrammatic illustration of which is shown in Fig. 1.8.2a, has been drawn by taking indication from Prabudiansyah et al. (2015), the study describing SecA1 and SecA2 interaction in $M t b$.

TAT is another system, independent of Sec, which translocates folded proteins. Its member proteins have similar N-terminal sequence, with specialty of double arginine motif (Frain et al. 2019). It has been hypothesized that they are involved in pathogenesis of TB by directly acting on host cell membrane and disturbing its signaling or release some lipid moieties that are consumed by Mtb (Muñoz-Elías et al. 2006). Rv2525c is the member of TAT system, and is highly virulent for mice (Saint-Joanis et al. 2006). This protein has structural homology with transglycosidase enzyme involved in bacterial wall synthesis (Kelley et al. 200o). Organization of TAT genes in $M t b$ and its comparison with E. coli has 
been shown in Fig. 1.8.2b. This figure has been strategized by taking notion from Saint-Joanis et al. (2006) research. The third category of secreting proteins i.e. T7SS/Esx is a very special type; its proteins are smaller in size and lacking secretory signal (Sorensen et al. 1995). Most of the proteins in this system are functionally hypothetical. The two common members are ESAT-6 and CFP-10, which are considered immunodominant antigens (Wards et al. 200o). The whole Esx family has two novel features; 1) all member proteins have almost 100 amino acids sequence with Trp-X-Gly (WXG) motif in the center of protein that gives helix turn helix structure (Renshaw et al. 2005), 2) transmembrane proteins with FtsK-SpoIIIE-like ATPase family (Pallen 2002). Fig. 1.8.2C demonstrates the organization of Esx system, designed as per concept taken from the study of Delogu et al. (2013).

Specialized protein transport mechanism has been used by $M t b$ to deliver protein in its inner and outer membrane. Type VII secretion system $\left(\mathrm{T}_{7} \mathrm{SS}\right)$ is the one among transport systems that was identified in 2003 in Mtb. A total of $5 \mathrm{~T}_{7} \mathrm{SS}$ systems have been recognized in $M t b$ and commonly designated as Esx-1, Esx-2, Esx-3, Esx4 and Esx-5 (Gröschel et al. 2016). Almost all Esx have common features, including small secreted protein pair (each of $\sim 100$ amino acids) with conserved Trp-X-Gly (WXG) motif (Vaziri and Brosch 2019). This helps the secretory pair to make helix-turn-helix conformation. This system also has some conserved transmembrane proteins that help in the secretion of secretory pair, hence called Esx conserved components (Ecc) (Famelis et al. 2019). The idea of plotting Fig. 1.8.2d has been taken from the Houben et al. (2014) manuscript; designed and entitled "Take five-Type VII secretion systems of Mycobacteria".

\section{Conclusion}

Tuberculosis (TB) is a highly contagious disease of human beings and bovines, caused by Mycobacterium tuberculosis (Mtb) and Mycobacterium bovis (M. bovis), respectively. Both species share almost 99\% genomic identity and can infect each other's host with less severity of disease than infecting their own host. This observation leads to the development of Bacillus Calmette-Guérin (BCG) vaccine (a modified form of $M$. bovis) for human TB. Currently, BCG is the only available vaccine for TB; it offers no booster and efficacy in adults. The promising adjunct or BCG complement remains major challenge to overcome TB menace. Potent vaccine against $M t b$ that can be used safely in adults and also as a booster is still in question, even after 100 years discovery of BCG. Therefore, exploring the new vaccine candidates for TB vaccine development is the need of time and this can be done by researching more on epidemiology and function genetics of $M t b$.

\section{REFERENCES}

Ameni G et al., 2011. Mycobacterium tuberculosis infection in grazing cattle in central Ethiopia. The Veterinary Journal 188: 359-361.
Ameni G, 2013. Transmission of Mycobacterium tuberculosis between farmers and cattle in central Ethiopia. PLoS One 8: e76891.

Andersen $\mathrm{P}$ and Doherty TM, 2005. The success and failure of BCG-implications for a novel tuberculosis vaccine. Nature Reviews Microbiology 3: 656-662.

Ayele WY et al, 2004. Bovine tuberculosis: An old disease but a new threat to Africa. The International Journal of Tuberculosis and Lung Diseases 8: 924- 937.

Behera D, 2010. Textbook of Pulmonary Medicine. Jaypee Brothers Medical Publishers Pvt. Limited.

Bellini C and Horváti K, 2020. Recent advances in the development of protein-and peptide-based subunit vaccines against tuberculosis. Cells 9: 2673.

Berg S et al., 2009. The burden of mycobacterial disease in Ethiopian cattle: Implications for public health. PloS One 4: e5068.

Bhardwaj A, 2014. Dissecting the enigma of Mycobacterium tuberculosis pathogenesis. Science Translational Medicine 6: 244ec116-244ec116.

Bohlin J et al., 2019. Estimation of AT and GC content distributions of nucleotide substitution rates in bacterial core genomes. Big Data Analytics 4: 1-11.

Brosch R et al., 1998. Use of a Mycobacterium tuberculosis $\mathrm{H}_{37} \mathrm{Rv}$ bacterial artificial chromosome library for genome mapping, sequencing and comparative genomics. Infection and Immunity 66: 2221- 2229.

Brosch R et al., 2002. A new evolutionary scenario for the Mycobacterium tuberculosis complex. Proceeding of the National Academy of Sciences of the United States of America 99: 3684-3689.

Calmette A et al., 1927. La vaccination préventive contre la tuberculose par le" BCG," Masson et cie 1927.

Cheigh CI et al., 2010. Post-treatment reactivation of tuberculosis in mice caused by Mycobacterium tuberculosis disrupted in mceiR. Journal of Infectious Diseases 202: 752-759.

Choi SY et al., 2018. Vaccine potential of ESAT-6 protein fused with consensus CD4+ T-cell epitopes of PE/PPE proteins against highly pathogenic Mycobacterium tuberculosis strain HN878. Biochemical and Biophysical Research Communications 503: 2195-2201.

Cole ST et al., 1998. Deciphering the biology of Mycobacterium tuberculosis from the complete genome sequence. Nature 396: 190-190.

Crampin AC et al., 2009. What has Karonga taught us? Tuberculosis studied over three decades. The International Journal of Tuberculosis and Lung Disease 13: 153-164.

Daniel TM, 2006. The history of tuberculosis. Respiratory Medicine 100: 1862-1870.

Davies P, 2006. Tuberculosis in humans and animals: Are we a threat to each other? Journal of the Royal Society of Medicine 99: 539-540.

Delogu G et al., 2013. The biology of Mycobacterium tuberculosis infection. Mediterranean Journal of Hematology and Infectious Diseases 5: e2013070.

DiGiuseppe CPA and Cox JS, 2007. Protein secretion systems in Mycobacteria. Cellular Microbiology 9: 1376-1384. 
Dhivya S et al., 2018. An immunoinformatics approach to define $\mathrm{T}$ cell epitopes from polyketide and nonribosomal peptide synthesis proteins of Mycobacterium tuberculosis as potential vaccine candidates. Journal of Molecular Recognition 31: e2685.

Famelis $\mathrm{N}$ et al., 2019. Architecture of the mycobacterial type VII secretion system. Nature 576: 321-325.

Frain KM et al., 2019. Transport of folded proteins by the Tat system. The Protein Journal 38: 377-388.

Fritsche A et al., 2004. Mycobacterium bovis tuberculosis: From animal to man and back. The International Journal of Tuberculosis and Lung Disease 8: 903-904.

Glaziou P et al., 2013. Global epidemiology of tuberculosis. In: Seminars in Respiratory and Critical Care Medicine. Thieme Medical Publishers; pp: oo3016.

Glaziou P et al., 2015. Global epidemiology of tuberculosis. Cold Spring Harbor Perspectives in Medicine 5: ao17798.

Glick BR et al., 2014. Medical Biotechnology. Washington DC: ASM Press; pp: 632-663.

Gong $\mathrm{W}$ et al., 2021. Peptides-based vaccine $\mathrm{MP}_{3} \mathrm{RT}$ induced protective immunity against Mycobacterium tuberculosis infection in a humanized mouse model. Frontiers in Immunology 12: 1393.

Golden MP and Vikram HR, 2005. Extrapulmonary tuberculosis: An overview. American Family Physician 72: 1761-1768.

Gröschel MI et al., 2016. ESX secretion systems: Mycobacterial evolution to counter host immunity. Nature Reviews Microbiology 14: 677-691.

Gutierrez MC et al., 2005. Ancient origin and gene mosaicism of the progenitor of Mycobacterium tuberculosis. PLoS Pathogen 1: e5.

Hackendahl NC et al., 2004. Putative transmission of Mycobacterium tuberculosis infection from a human to a dog. Journal of the American Veterinary Medical Association 225: 1573-1577.

Philipp WJ et al., 1996. An integrated map of the genome of the tubercle bacillus, Mycobacterium tuberculosis $\mathrm{H}_{37} \mathrm{Rv}$, and comparison with Mycobacterium leprae. Proceedings of the National Academy of Sciences of the United States of America 93:3132-3137.

Hesseling AC et al., 2007. The risk of disseminated Bacille Calmette-Guerin (BCG) disease in HIV-infected children. Vaccine 25: 14-18.

Hirsh AE et al., 2004. Stable association between strains of Mycobacterium tuberculosis and their human host populations. Proceedings of the National Academy of Sciences of the United States of America 101: 48714876.

Hossain $\mathrm{M}$ et al., 2017. Computational identification and characterization of a promiscuous T-cell epitope on the extracellular protein $85^{\mathrm{B}}$ of Mycobacterium spp. for peptide-based subunit vaccine design. BioMed Research International 2017: Article \# 4826030.

Houben ENG et al., 2014. Take five- Type VII secretion systems of Mycobacteria. Biochimica et Biophysica Acta. Molecular Cell Research 1843: 1707-1716.
Jose L et al., 2016. Hypothetical protein Rv3423.1 of Mycobacterium tuberculosis is a histone acetyltransferase. The Federation of the European Biochemical Societies Journal 283: 265-281.

Kaufmann SH, 2014. Tuberculosis vaccine development at a divide. Current Opinion in Pulmonary Medicine 20: 294-300.

Kaufmann SH and Parida SK, 2007. Changing funding patterns in tuberculosis. Nature Medicine 13: 299-303.

Kelley LA et al., 200o. Enhanced genome annotation using structural profiles in the program $3 \mathrm{D}$-PSSM. Journal of Molecular Biology 299: 499-520.

Khatoon $\mathrm{N}$ et al., 2017. Exploring Leishmania secretory proteins to design $\mathrm{B}$ and $\mathrm{T}$ cell multi-epitope subunit vaccine using immunoinformatics approach. Scientific Reports 7: 1-12.

Klettner CA et al., 2012. The effect of turbulence on the spreading of infectious airborne droplets in hospitals. New Approaches in Modeling Multiphase Flows and Dispersion in Turbulence, Fractal Methods and Synthetic Turbulence. Springer Dordrecht; pp: 141-152.

Kumar V et al., 2007. Robbins Basic Pathology. Saunders Elsevier (2003); pp: 718-721.

Lenz LL et al., 2003. SecA2-dependent secretion of autolytic enzymes promotes Listeria monocytogenes pathogenesis. Proceedings of the National Academy of Sciences of the United States of America 100: 12432-12437.

Lindenstrøm $\mathrm{T}$ et al., 2017. T cells primed by live Mycobacteria versus a tuberculosis subunit vaccine exhibit distinct functional properties, EBioMedicine 27: 27-39.

Loddenkemper R et al., 2015. Clinical aspects of adult tuberculosis. Cold Spring Harbor Perspectives in Medicine 6: ao17848.

Long $\mathrm{R}$ and Schwartzman K, 2014. Pathogenesis and transmission of tuberculosis. Canadian Tuberculosis Standards, $7^{\text {th }}$ Edition. Public Health Agency of Canada.

Ma J et al., 2017. A multistage subunit vaccine effectively protects mice against primary progressive tuberculosis, latency and reactivation. EBioMedicine 22: 143-154.

MacNeil A et al., 2019. Global epidemiology of tuberculosis and progress toward achieving global targets-2017. Morbidity and Mortality Weekly Report 68: 263 .

Majlessi L et al., 2015. Release of mycobacterial antigens. Immunological Reviews 264: 25-45.

Messenger AM et al., 2014. Reverse zoonotic disease transmission (zooanthroponosis): a systematic review of seldom-documented human biological threats to animals. PloS One 9: e89055.

Muñoz-Elías et al., 2006. Role of the methylcitrate cycle in Mycobacterium tuberculosis metabolism, intracellular growth and virulence. Molecular Microbiology 6o: 1109-1122.

Pallen MJ, 2002. The ESAT-6/WXG10o superfamily and a new Gram-positive secretion system? Trends in Microbiology 10: 209-212. 
Parsons LM et al., 2002. Rapid and simple approach for identification of Mycobacterium tuberculosis complex isolates by PCR-based genomic deletion analysis. Journal of Clinical Microbiology 40: 2339-2345.

Prabudiansyah I et al., 2015. In vitro interaction of the housekeeping SecA1 with the accessory SecA2 protein of Mycobacterium tuberculosis. PloS One 10: e0128788.

Quigley J et al. 2017. The cell wall lipid PDIM contributes to phagosomal escape and host cell exit of Mycobacterium tuberculosis. MBio 8: eoo148-17.

Renshaw PS et al., 2005. Structure and function of the complex formed by the tuberculosis virulence factors CFP-10 and ESAT-6. The EMBO Journal 24: 2491-2498.

Rockwood RR, 2007. Extra-pulmonary TB: What you need to know. The Nurse Practitioner 32: 44-49.

Saint-Joanis B et al., 2006. Inactivation of Rv2525c, a substrate of the twin arginine translocation (Tat) system of Mycobacterium tuberculosis, increases $\beta$ lactam susceptibility and virulence. Journal of Bacteriology 188: 6669-6679.

Sharma G et al., 2015. The interaction of mycobacterial protein Rv2966c with host chromatin is mediated through non $\mathrm{CpG}$ methylation and histone $\mathrm{H}_{3} / \mathrm{H}_{4}$ binding. Nucleic Acids Research 43:3922-3937.

Sorensen AL et al., 1995. Purification and characterization of a low-molecular-mass T-cell antigen secreted by Mycobacterium tuberculosis. Infection and Immunity 63: 1710-1717.

Sreevatsan S et al., 1997. Restricted structural gene polymorphism in the Mycobacterium tuberculosis complex indicates evolutionarily recent global dissemination. Proceedings of the National Academy of Sciences of the United States of America 94: 98699874 .
Vaziri F and Brosch R, 2019. ESX/Type VII secretion systems: An important way out for Mycobacterial proteins. Protein Secretion in Bacteria 7: 351-362.

Villarreal-Ramos B et al., 2018. Experimental infection of cattle with Mycobacterium tuberculosis isolates shows the attenuation of the human tubercle bacillus for cattle. Scientific Reports 8: 1-13.

Waksman SA, 1964. The Conquest of Tuberculosis. Berkeley: University of California Press, USA.

Wang J et al., 2017. The mycobacterial phosphatase PtpA regulates the expression of host genes and promotes cell proliferation. Nature Communications 8: 1-16.

Wards BJ et al., 200o. An ESAT6 knockout mutant of Mycobacterium bovis produced by homologous recombination will contribute to the development of a live tuberculosis vaccine. Tubercle and Lung Disease 80: 185-189.

World Health Organization, 2014. Antimicrobial resistance: 2014 global report on surveillance. World Health Organization.

World Health Organization, 2018. Global tuberculosis report 2018. Geneva, Switzerland: World Health Organization.

World Health Organization, 2019. Global tuberculosis report 2019. Geneva, Switzerland: World Health Organization.

Yaseen I et al., 2015. Mycobacteria modulate host epigenetic machinery by Rv1988 methylation of a non-tail arginine of histone $\mathrm{H}_{3}$. Nature Communications 6: 1-13.

Yates TA et al., 2016. The transmission of Mycobacterium tuberculosis in high burden settings. The Lancet Infectious Diseases 16: 227-238.

Zhu B et al., 2018. Tuberculosis vaccines: Opportunities and challenges. Respirology 23: 359-368. 